\title{
Community placement and reintegration of service users from long-term mental health care facilities
}

\author{
Bernard Janse van Rensburg \\ Division of Psychiatry, Department of Neurosciences, University of the Witwatersrand, Johannesburg, South Africa
}

\begin{abstract}
Objective: To ascertain community placement and reintegration of service users from long-term mental health care facilities. Method: This study reviewed the progress during 2003 with the alternative placement of a selected candidate group of 27 service users in some of Lifecare's long-term mental health care facilities in Gauteng. Results: Despite a significant amount of time and effort to find alternative options with family, old age homes or other non-governmental organizations, only $\mathrm{g}(33 \%)$ were placed a year later. A longer follow-up period however is necessary to ascertain the actual successful outcome of these placements. Conclusions: Concerns exist about sufficient development of community-based services in accordance with the new Mental Health Care Act (Act No. 17 of 2002), parallel to hospital-based care.
\end{abstract}

Keywords: Reintegration, Alternative placement, Long term mental health care, Policy, Legislation, Resources

Received: 31.01 .05

Accepted: 12.05 .05

\begin{abstract}
Introduction
Over the past decade, there have been policy changes on longterm inpatient mental health care in Gauteng Province and elsewhere in South Africa. These changes are reflected in new mental health legislation passed by parliament during 2002 in the form of the Mental Health Care Act, No 17 of $2002 .{ }^{1}$ The emphasis has shifted to appropriate community-based care, rehabilitation and reintegration into the community. As a result of these developments, pressure mounted on Lifecare, a large contracted private service provider to Gauteng's Department of Health, to continuously review its existing service users and to assess what proportion of them may be suitable for possible placement back into their community of origin. Lifecare is currently responsible for long term in-patient care, treatment and rehabilitation of mental health service users in several provinces of South Africa. During the study period in Gauteng alone, Lifecare had 3150 beds in 8 hospitals across the province. Lifecare's vision was stated, "to be a chosen partner for delivering and developing special health care services affordably, with excellence in quality". Admission - Principles that underpinned the admission criteria for long term care users to the different Lifecare facilities included: - that all admissions were effected in terms of Section 9 of the Mental Health Act, No 18 of 1973 (i.e. involuntary admissions); -
\end{abstract}

\section{Correspondence:}

Dr ABR Janse van Rensburg, Division of Psychiatry, Department of Neurosciences, 7 York Road, Parktown, 2193, Johannesburg, South Africa. email:ariane@zweb.co.za that the user had to reside in the province of Gauteng and/or the original reception order had to be issued by an magisterial district in Gauteng; - that the user must have been admitted to a provincial hospital with the same psychiatric disorder on at least three previous occasions in addition to the condition being chronic for at least 2 years; - that users had to be 25 years or older. An additional condition concerned the functionality of a service user, namely that a user had to demonstrate marked deterioration in functioning including self care, communication, appropriate expression of feelings, ability to sustain relationships, work performance and ability to participate in leisure activities, to the extent that the user is not able to live independently or be contained within family context. Continued detention - Until the implementation of the new mental health legislation, all service users of Lifecare's facilities were routinely admitted as involuntary ("certified") service users in terms of Section 9 of the old act (Mental Health Act, No. 18 of 1973). However, continued detention of involuntary service users following an initial period of 42 days had to be justified by subsequent annual, bi-annual and later tri-annual periodical reports on a user's mental state by the psychiatrist and medical superintendent to the Department of Health (DOH). The DOH in its turn issued instructions about the continued detention of service users, based on the submitted periodical reports. When a periodical report had to be compiled by the psychiatrist responsible, the following were generally considered: - any existing instructions by DOH following on previous periodical reports; - the progress of the service user since the last report; and - the current mental state of the service user in question. The periodical report would also include a 
statement about the diagnosis and the current medication used by the service user. When submitted, the $\mathrm{DOH}$ will again review the recommendations and issue a new set of instructions about further detention. Discharge - With regard to the possible discharge of service users, the following were considered: - if the service user still qualified to be an involuntary service user (according to Section 9 of the Mental Health Act, No. 18 of 1973); - the service user's general functioning; and - family circumstances or other placement options.

\section{Method}

A survey was conducted during January 2003 to February 2004 of current service users $(n=738)$ in sections of three Lifecare facilities located in eastern Gauteng with a total of about 1000 beds, to identify candidates for possible discharge and alternative placement. Service users $(n=152)$ in Struisbult, Waverley, and female wards of Witpoort Care Centers, who were due for routine psychiatric reports in January to March 2003 on their mental health status and for recommendations to the $\mathrm{DOH}$ regarding their further detention in the specific facility, were reviewed by the psychiatrist with the assistance of the multi-disciplinary team (MDT). The study was conducted in three steps:

i. Identifying a candidate group for possible discharge and alternative placement from those due for a periodical report during January to March 2003;

ii. Compiling a demographic and clinical profile of the group selected; and

iii. Assessing progress with placement of the identified group 1 year later (February 2004).

Identifying the candidate group

While the specified criteria for admission, continued detention and discharge were taken into account as far as possible, no structured method of rating these factors was readily available for inclusion in these routine assessments. However, a potential candidate group was identified based on: - existing instructions by the DOH; - review by MDT; and - an updated mental state assessment. Possible options after discharge for alternative placement as voluntary or consent users (according to Sections 3 and 4 of the old Act) with family, an old age home or another non governmental organization (NGO) facility, were also considered according to a service user's area of origin and age. Compiling a demographic and clinical profile An analysis was made of the candidate group's age, gender, length of stay, diagnosis, area of origin, record of family contact and of existing instructions made to date by the DOH on their continued detention.

Assessing progress with placement

The potential candidate group was followed up one year later (February 2004) to assess the progress with alternative placement of these service users in the community. A review was done at this stage of: - what type of placement was originally considered; - the "resource units" (time and cost) used in the attempt to place them; - the area and facility where eventually placed; and - if the placement could regarded as successful. A placement would be considered to be successful if no problems (e.g. behavioral, relapse, compliance, access to medication) were reported to the social worker during a three-month follow-up period after discharge. "Resource units" used in the attempted placement, were established by considering time (of the social worker involved) spent on telephone calls, report writing and home/facility visits, as well as cost (telephone, transport, basic hourly rate for employment of social worker). A "resource unit" will therefore consist of the effort (time and cost) to attempt a successful placement. One "time unit" was calculated as follows: time on telephone calls average $20 \mathrm{~min}$ x 3 ( 1 hour), plus 1 hour report writing, plus 2 hours/visit x 2 (4 hours), which equals 6 hours. One "cost unit" (for services within $50 \mathrm{~km}$ range) consisted of: telephone cost at estimated R30/hour (R30.00), plus hourly rate of social worker R60x6 (R360.00), plus 2 return visits of $100 \mathrm{~km}$ each $x \mathrm{R} 1.35 / \mathrm{km}$ (R270.00), which equals R660.00.

\section{Results}

Identifying the Candidate Group - A total of 738 consultations was conducted during this period, of which 152 were for periodical report reviews due during January to March 2003 and 219 for six-monthly clinical reviews. Of the total 152 periodical reports due during January to March 2003, 27 (17.8\%) were included in the potential candidate group for possible alternative placement: 4 from Waverley, 1 from Witpoort and 22 from Struisbult.

The high number identified for possible discharge in Struisbult was not surprising, as a proportion of relatively higher functioning service users were transferred there from other Lifecare facilities in preceding months, to be included in a preparatory pre-discharge program.

\section{Profile of the group}

The ages of the group for possible placement ranged from 10 to 87 years:

\begin{tabular}{|l|l|l|l|l|l|l|l|l|}
\hline $\begin{array}{l}10-19 \\
\text { (years) }\end{array}$ & $20-29$ & $30-39$ & $40-49$ & $50-59$ & $60-69$ & $70-79$ & $80-89$ & TOTAL \\
\hline 1 & 1 & 4 & 5 & 8 & 6 & 1 & 1 & 27 \\
\hline $3.7 \%$ & $3.7 \%$ & $14.8 \%$ & $18.5 \%$ & $29.6 \%$ & $22.2 \%$ & $3.7 \%$ & $3.7 \%$ & $100 \%$ \\
\hline
\end{tabular}

\section{Gender}

8 were female and 19 were male. Length of stay for the group ranged from 1 to 38 years, with the average length of stay (i.e. the average time since the reception order was issued) of 14.8 years. Diagnoses included:

\begin{tabular}{|l|l|l|}
\hline Schizophrenia & 12 & $44.4 \%$ \\
\hline Schizo-affective Disorder & 4 & $14.8 \%$ \\
\hline Mental Retardation & 6 & $22.2 \%$ \\
\hline Dementia & 4 & $14.8 \%$ \\
\hline Bipolar Mood Disorder & 1 & $3.7 \%$ \\
\hline TOTAL & 27 & $100 \%$ \\
\hline
\end{tabular}

\section{Family contact}

$12(44.4 \%)$ had no contact with family since the last periodical report (between 1-3 years); 11 (40.7\%) had regular contact and $4(14.8 \%)$ seldom. District of origin: 10 of the 27 (48.2\%) were from areas outside of Gauteng and 3 (11\%) not stated. Existing instructions from the $\mathrm{DOH}$ with regard to the identified candidate group assessed during January to March 2003, included: - "further detention"; - "discharge"; - "requesting a further report"; or - were not documented. 


\section{Progress with placement}

Updated periodical reports was submitted to the $\mathrm{DOH}$ for all service users in the originally identified potential candidate group $(n=27)$ by the end of March 2003. While all of the 27 service users for possible discharge in the group were still severely dysfunctional according to the original admission criteria, on review of their status only 12 service users $(44.4 \%)$ were regarded by the MDT as suitable to be "decertified" and therefore, to be sent on a trial leave of absence with subsequent discharge and to be placed appropriately, in an alternative structured environment as a voluntary or consent service user (Sections 3 and 4 of the Mental Health Act, No 18 of 1973). The remaining 15 (55.6\%) were recommended to receive further care and supervision in a Lifecare facility. In response to these updated periodical reports and recommendations, the $\mathrm{DOH}$ however instructed subsequently the discharge of $18(68 \%)$ of the original 27 , requested further reports on $5(18 \%)$ and only approved the further detention of 4 (14\%).

Potential alternative placement options for the original candidate group of 27 service users regarded to be most suitable by the MDT, included: with family, $n=8(29.6 \%)$; in an old age home, $n=6(22.2 \%)$; or with another non-governmental organization, $\mathrm{n}=13(48.2 \%)$. However, on reassessment of the whole process in February 2004, which started with 27 potential service users in the original candidate group for possible discharge, which was then reduced to 12 as recommended in the updated periodical reports by the psychiatrist and then increased again to 18 as instructed by the $\mathrm{DOH}$, four $(15 \%)$ of these service users (in Struisbult Care Center) were ruled out before any process of placement could start, because of their subsequent unstable mental state or relapse during the second part of 2003. One service user (4\%) in the original candidate group died during the one-year follow-up period and two $(8 \%)$ were transferred to other Lifecare facilities in Gauteng. For eleven $(40 \%)$ of the original 27 service users, no real arrangements for alternative placement were commenced with and one year after initial assessment, they were still in Struisbult Care Center. By February 2004, only 9 (33\%) of the 27, or $50 \%$ of the 18 subsequently instructed by the DOH to be discharged, had eventually been placed. Of these 9 service users, 3 were placed in OAH's, 2 with family, and 4 with other NGO's.

Geographically, 8 of these users were eventually placed in Gauteng and 1 was placed in his district of origin in the Northern Cape Province. With regard to the success of placement, some of the users were discharged within the three months prior to the one-year follow-up period in February 2004. It was therefore too early to assess the outcome of their placement. Of those placed earlier, one was still being followed up for behavior problems experienced and another placed in eastern Gauteng with his family, had gone missing. "Resource units" used in the attempt to consider and commence with the placement of the original 27 service users in the candidate group, were estimated to be: 0 (in the case of 9 users), 0,5 (3 users), 1 (8 users), 2 (5 users), 3 (1 user) and 4 (1 user). This amounted to a total of 26.5 "resource units" and therefore total "time units" of 159 hours (26.5x6hours) spent on efforts to attempt successful placement for members of this group. It represented an average of 5.9 hours spent per patient and amounted in total to about 20 (8-hour) weekdays. With regard to cost according to this calculation, total "cost units" of efforts to find alternative placement for this study group amounted to R17 490.00 (26.5xR660.00), with an average cost per service user of R647.80.

\section{Discussion}

The results of this study highlighted in the first place the difficulty to select, prepare and anticipate according to current review processes, the successful placement of long-term service users in an appropriate alternative setting. Although admission and discharge criteria and factors contributing to being an involuntary, assisted or voluntary user had been considered, no structured measure of these could readily be implemented to ensure a specific outcome. Factors such as: individual insight and functionality; compliance; dependence; the course of illness and recurrence of symptoms; length of stay in hospital; family and community resources; and the service provision infrastructure impacted on the successful outcome of a long-term service user in the community. Reasons for unsuccessful placement of long-term mental health care users in this study included the service users' chronic and unstable status to begin with and their subsequent relapse during the one-year study period. Another reason was that the NGOs approached during the study period, often excluded individuals older than 40 years, while old age homes (OAHs) usually only took people older than 60 years. This resulted in having very limited placement options for somebody in this study population between 40-60 years of age, unless their families could or would accept them. Reasons for the rejection by NGO's or OAH's of applications made, included the fact that applicants from this group had a psychiatric diagnosis and required additional supervision and care and that certain psychiatric drugs were allegedly not available at the facilities. In two cases, service users were placed for a while, but were returned to Lifecare because of alleged theft of clothes in one instance and in the other, rejected because of perceived "promiscuous" behaviour. Families mainly refused placement with them due to their restricted emotional, physical and financial means to accept responsibility for their relative. The time and cost to place and reintegrate service users into the community from long-term mental health care facilities as alluded to by this study, represented a significant proportion of existing human resources available within Lifecare during the time of the study. In addition, a daily rate per service user in a long-term facility such as Lifecare's care centres, must also be taken into account when calculating costs incurred by over-extended inpatient stay.

Studies in a review of recent literature, reported on the reintegration of mental health service users in the community after extended periods of inpatient stay in Europe ${ }^{2-9}$, the $\mathrm{UK}^{4-}$ ${ }^{5}$, North America ${ }^{6,7}$, Australia $^{8}$ and South America9 ${ }^{9}$ These studies highlight particular shortcomings and certain preconditions in the international experience. Most of the studies emphasized the need to develop community-based facilities parallel and preferably prior to the closure or reduction of inpatient capacity. Results where adequate community-based services were not developed included increased admission rates to general hospitals, more frequent relapses, homelessness and criminalization of mental health services users. Three types of cooperative arrangements were compared in Holland: a psychiatric hospital renting a unit in a residential home for 
the elderly, a psychiatric hospital stationing metal health professionals in a residential home on a permanent basis, and a residential home employing its own psychiatrically trained staff. $^{2}$ The most promising model appeared to be the second option, where mental health professionals were assigned to a residential home remaining administratively and operationally distinct from the standard residential services. The discharge of 4492 service users from 22 public psychiatric hospitals during 1994 and 2000 in Italy was reviewed and it was concluded that the potential risk of abandonment due to deinstitutionalization was not observed in this population, as they were discharged to highly supervised settings. ${ }^{3}$ The care for a British group of 72 long-stay psychiatric service users regarded as unsuitable for community placements was investigated $^{4}$ and even from this group, who generally demonstrated minimal change in functioning and social behavior over 5 years, $40 \%$ were finally to be resettled in various care homes after "slow-stream" rehabilitation within specialized facilities. Goldberg argues that for care in the community to succeed, there must be adequate numbers of (in patient) beds available, a range of sheltered residential accommodation in the community, as well as enough staff to provide a service for them. ${ }^{5}$ A multi-country comparison on health and mental health reform used a policy framework to compare developments in Canada, the United States, Britain and Australia. ${ }^{6}$ From this review, themes emerged including that although the reforms in all these countries espouse a progressive community-based philosophy of care, in most cases this is rhetorical as the real agenda is cost containment. Either very little or insufficient reallocation of resources to community care has occurred. Cost concerns were also found to be driving deskilling and deprofessionalization in mental health care. Lamb and Bachrach observed that community mental health care is potentially more humane and more therapeutic than hospital care, but this potential is realized only when certain preconditions have been met. ${ }^{7}$ These preconditions include: realizing that deinstitutionalization is a social process with secondary consequences, tailoring service planning to individual needs, facilitating access to hospital care, ensuring cultural relevance of services, involving mentally ill persons in service planning, maintaining flexibility of services and continuity of care in the community. A report on 20-bed community care units in Australia, built in suburban locations and staffed by multidisciplinary clinical teams on a 24 hour basis, concluded that these units were developed to provide accommodation, clinical care and rehabilitation of patients discharged from long-stay open wards. ${ }^{8}$ A review of the transition in South American countries from a system based on the large psychiatric hospital to a range of alternative structures, commented that it was accompanied by several economic, social and political changes. ${ }^{9}$ Intensive efforts had to be made to collect and disseminate information and to monitor the development and outcome of these programs.

Apart then from distinct and applicable selection criteria of potential long-term care candidates for possible discharge and reintegration, when compared to the international data, this limited experience of attempted placements of chronic mental health care users in a South African context also gave rise to certain reservations about the capacity of the mental health care system in South Africa. Concerns include issues such as: - sufficient parallel development of community-based services in view of the emphasis on community care by the new Mental Health Care Act ${ }^{1}$; adequate supervised facilities (e.g. day care centers) to accommodate service users discharged from long-term care facilities; availability of mental health professionals to provide services in alternative facilities or programs; overcoming of fragmentation of continued rehabilitation options after discharge; provision of adequate budget allocation for the implementation of policy; and the process to monitor the outcome of these policy developments.

\section{Conclusion}

The implication of the study experience is that attention must be given to: - appropriate guidelines, standards and measures to identify possible candidates for successful discharge from long-term care communities; and to - care scenarios, conditions and opportunities such as extended options for structured and supervised residential and day care of long-term mental health care service users in the community. At the same time, admission criteria to Lifecare facilities should be strictly adhered to in order to reduce and eliminate inappropriate admissions that later on will have to be relocated if unsuitable. The new legal dispensation opened the opportunity and responsibility for public and private role-players in mental health care service provision, to apart from involuntary hospital based care for long-term service users, also develop innovative care packages including assisted and voluntary community based programs.

\section{References}

1. Mental Health Care Act, Act No. 17 of 2002.

2. Depla MFIA, Pols J, De Lange J, Smits CHM, De Graaf R, Heeren TJ. Integrating mental health care into residential homes for the elderly: an analysis of six Dutch programs for older people with severe and persistent mental illness. J Am Geriatr Soc 2003; 51(9): 1275-9.

3. D'Avanzo B, Barbato A, Barbui C, Battino RN, Civenti G, Frattura L. Discharges of patients from public psychiatric hospitals in Italy between 1994 and 2000. Int J Soc Psychiatry 2003 Mar; 49(1): 27-34.

4. Trieman N, Leff J. Long-term outcome of long-stay psychiatric inpatients considered unsuitable to live in the community. Br J Psychiatry 2002; 181:428-32.

5. Goldberg D. The future pattern of psychiatric provision in England. Eur Arch Psychiatry Clin Neurosci 1999; 249(3): 123-7.

6. Shera W, Aviram U, Healy B, Ramon S. Mental health system reform: a multi country comparison. Soc Work Health Care 2002; 35(1-2): 547-75.

7. Lamb HR, Bachrach LL. Some perspectives on deinstitutionalization. Psychiatr Serv 2001; 52(8): 1039-45.

8. Trauer $T$, Farhall J, Newton $R$, Cheung P. From long-stay psychiatric hospital to Community Care Unit: evaluation at 1 year. Soc Psychiatry Psychiatr Epidemiol 2001; 36(8): 416-9.

9. Larrobla C, Botega NJ. Restructuring mental health: a South American survey. Soc Psychiatry Psychiatr Epidemiol 2001; 36(5): 256-9. 